\title{
ON THE MILD AND WILD MECHANICAL ROUGHNESS OF THE LITHOSPHERE
}

\section{Sobre la suave y salvaje rugosidad mecánica de la litósfera}

\section{Gerd Gudehus}

Institute of Soil Mechanics and Rock Mechanics, Karlsruhe Institute of Technology, Karlsruhe, Germany, gerd.gudehus@ibf.uni-karlsruhe.de

The spatio-temporal mechanical fractality of the lithosphere can be represented by means of Lévy-statistics and fractional calculus. This novel concept is outlined with geometrical and energetic arguments. The fractality originates from the pore system and appears in displacements, stresses and seismicity. It is shown that the roughness may be neglected for the stable range, but not for critical phenomena at the verge of energetic convexity. The outline leads from rough coastlines via sand samples to tectonic chain reactions. Equations and theorems are physically interpreted without algebra and proofs.

Keywords: fractality, lithosphere, roughness
Fecha de entrega: 12 de diciembre 2011 Fecha de aceptación: 20 de abril 2012
La fractalidad mecánica espacio-temporal de la litósfera puede ser representada por medio de la estadística de Lévy y cálculo fraccional. Este nuevo concepto es planteado con argumentos geométricos y energéticos. La fractalidad se origina del sistema poroso y aparece en desplazamientos, tensiones y sismicidad. Se muestra que la rugosidad podría no ser considerada en el rango estable, pero esto no es posible en fenómenos críticos al borde de convexidad energética. Este planteamiento cubre bordes costeros rugosos, muestras de arena y reacciones tectónicas en cadena. Ecuaciones y teoremas son interpretados fisicamente sin la necesidad de álgebra ni pruebas.

Palabras clave: fractalidad, litósfera, rugosidad

\section{Introduction}

The external fractality of the lithosphere was discovered by Mandelbrot (1982). Rough coastlines can be reproduced in hatched magnifications of sections. The log-log plot of the minimum number $N$ of squares needed to pave a coastline (Figure 1a) with squares versus their size $d$ is a straight line as shown in Figure 1b. This means $N=N_{r}\left(d_{r} / d\right)^{y}$ with reference values $N_{r}, d_{r}$ and a fractal dimension $1<\gamma<$ 2. The self-similarity holds within bounds or cutoffs $d_{\text {min }}$ and $d_{\max }$. The coastline length is thus $l \approx N_{r} d_{r}\left(d_{r} / d\right)^{r-1}$. The rough surface of a landscape can exhibit a similar fractality (Figure 2a). The minimum number $N$ of cubes of size $d$ needed to enclose the surface is $N=N_{r}\left(d_{r} / d\right)^{2 \gamma}$ if plane cross sections exhibit $\gamma$ as above. The area is thus $A \approx N_{r}$ $d_{r}^{2}\left(d_{r} / d\right)^{2(\gamma-I)}$. Length and area are no more extensive as without roughness, i.e. for $\gamma=1$.

A rough line or surface is called monofractal if its $\gamma$ is the same everywhere, otherwise multifractal. This concept works likewise for time-dependent evolutions observed at the surface of the lithosphere, e.g. seismograms of points or hydrograms of rivers. Mandelbrot (1999) uses the rather poetic expression mild and wild roughness in case of mono or multifractality, respectively. These notions may be taken over to spatially and temporally fractal distributions of displacements and forces, i.e. to mechanics with roughness. This requires an objective definition of gradients and time rates although rough distributions versus site $x_{i}$ and time $t$ have no unique tangents. Corresponding objective integrals over $x_{i}$ and $t$ are needed for balances of classically extensive quantities, viz. mass, energy and momentum or parts of them.

Mandelbrot knew of course that landscapes are shaped by tectonics, erosion and sedimentation, but his algorithms produce at best look-alikes with roughness and do not represent mechanisms. He attributed multifractals to 'multiplicative cascades', but this again is mere mathematics and not yet physics. Humid sand surfaces, e.g. in a box or at the beach (Figure 2b), can exhibit fractality, and may substitute theoretical physical models with roughness. Scaling up model test results to bigger sizes and longer times requires a dimensional backbone from 
physical theories. This is not supplied by the classical rules of similarity in case of fractality.

a)

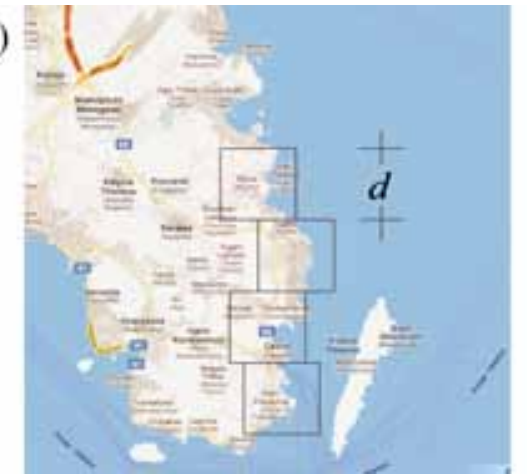

b)

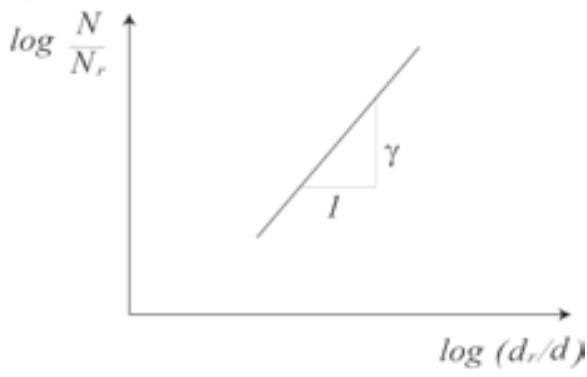

Figure 1: a) Boxes of size $d$ covering a coastline (Attica, Greece) and b) number of boxes versus reciprocal value of their size (qualitative)

The internal fractality of lithosphere sections - or of smallscale substitutes - is not as thoroughly visible as the external one, boreholes and sensors give at best fragmentary hints. Natural pore systems $(\pi \circ \rho \circ \varsigma=$ passage $)$ appear to be fractal. Their monofractality can be explained by means of rather fictitious data (see in the next section). Systems of tectonic faults, which are more porous than their vicinity, exhibit self-similarity in hatched sections. This kind of fractality is likewise observed in model tests and hypoplastic simulations as shown in Figure 3 (Gudehus 2011). The non-uniformity of groundwater flow points also to fractal pore systems (the permeability is proportional to the square of the pore size). It appears that the complete lithosphere, i.e. from its free surface down to the Moho discontinuity, has a fractal pore system, of course with lower pore fractions for higher pressures and temperatures.

Spatial distributions of internal pressures and stresses exhibit likewise fractality. This force-roughness plays already a role for soil samples, when one tries to capture it by hidden variables like back stress or intergranular strain
(Gudehus 2011).

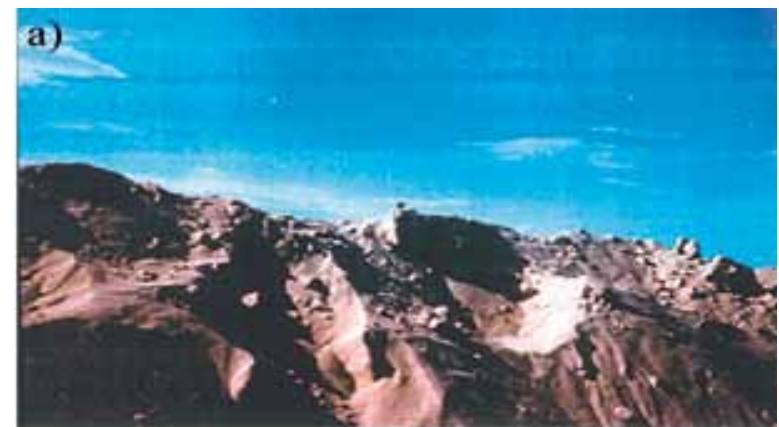

b)

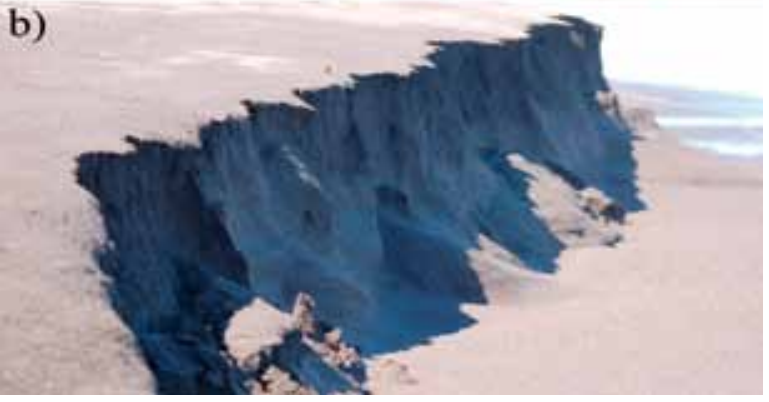

Figure 2: Fractal sand structures of $c a .0 .3 \mathrm{~m}$ height at the beach, a) mountain ridge (photo R. Gudehus) and b) cliff (photo M. Poblete)

a)

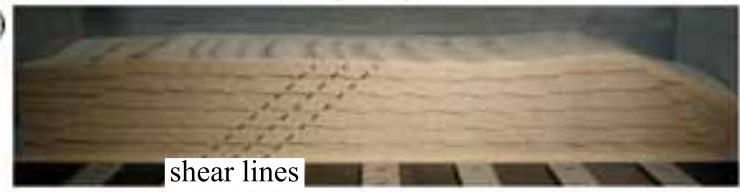

b)

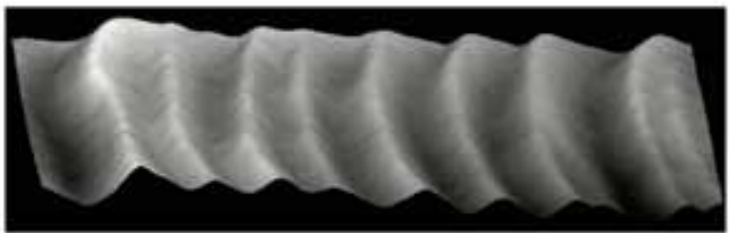

c)
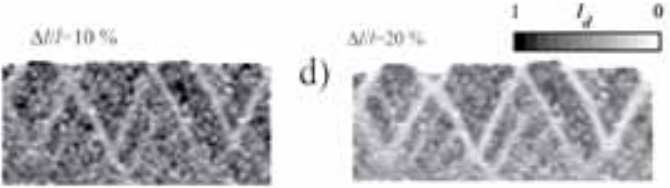

Figure 3: a), b) Patterns of shear bands in a model setup and c), d) in two steps of a simulation (Gudehus 2011)

Such approaches are not explicitly fractal, but tacitly scale-independent within reasonable bounds. The forceroughness is known from model tests and building sites with sand, it causes an indeterminacy of structural forces in or at the ground. It is more marked for rocks so that stresses at samples or structures in situ can hardly be determined. 
The world stress map of the lithosphere exhibits spatial fluctuations with wavelenghts from $c a .1 \mathrm{~km}$ to $1000 \mathrm{~km}$ (Heidbach et al. 2010). Stresses in the lithosphere are estimated by means of borehole measurements and plate tectonics. Several decades of fluctuation lengths suggest fractality, certainly multifractality in connection with which convection cycles of the asthenosphere impose some order to the lithosphere.

The force-roughness can be frozen for a while in stable sections of the lithosphere (what that means will be explained later on). Changes at boundaries - from above, below or aside - lead to changes of position and state of lithosphere sections or its small substitutes. Apart from smooth and rather slow changes due to thermal activation, such changes are jerky and accompanied by seismicity. The sound-roughness is audible as crackling of soil and rock samples, and more dramatically with breaking structures at or in the ground. Earthquakes exhibit similar emissions with lower frequencies due to bigger extensions. The spectra are often simplified as $1 / f$ noise, this notion is also used by Mandelbrot (1999) if the slope in a loglog spectrum diverges from -1 and indicates fractality. One may understand force-roughness as latent soundroughness, and would like to know when and how the latter arises.

The dynamics of the lithosphere is evidently multifractal and so complex that at best sections or substitutes of them can be captured by mechanical models. Initial states are inevitably partly subjective, and an additional indeterminacy arises from arbitrary boundaries separating near-fields from far-fields (Gudehus 2011). The interplay of thermal and seismic activations depends on space and time scales and it should not be separated from the fractality. Gain and loss of stability is evidently a key issue, but the treatment of it with fractality poses open questions. Critical phenomena with pattern formation and chaos for geo-matter were first considered with minute sand avalanches (Bak et al. 1987). Similar attempts were made with fractals in structural geology (Turcotte 1997) and rock mechanics (Xie 1993).

Recently, Gudehus and Touplikiotis (2012) proposed a more comprehensive approach by means of the fractional calculus, and a more mathematical paper on fractional stability is underway (Gorenflo et al. 2012). The present paper is less mathematical, however, it employs further physical arguments. First, the transition from fractal and Lévy-type distributions to fractional balances is outlined with the focus on geo-matter. Second, attenuation and subordination are introduced for fractionally uniform zones and indicated beyond. Third, the quasi-local loss of stability and then with chain reactions of increasing size are presented. Fourth, the interplay of seismic and thermal interactions is touched. Finally, conclusions with summary and outlook complete this more qualitative than quantitative paper.

\section{From fractals to fractional balances}

Consider the fractal pore system of an idealized layer as shown in Figure 4. Straight lines cut solid and void fractions in such a way that hatched magnifications exhibit a similar rough sequence (Figure 4a). The solid mass $m$ in a cube of size $d$ yields a straight log-log plot (Figure $4 \mathrm{~b}$ ) with reference values $m_{r}, d_{r}$ and a fractal dimension $c a$. $0.9<\beta<1$ instead of $\beta=1$ without roughness. This holds within cutoffs $d_{\min }$ near a rock fraction size and $d_{\max }$ below the layer thickness $h$. The solid fraction is thus $\left.n_{s}=n_{r}(d d)^{\beta}\right)^{\beta-1}$ for lines, $n_{s}=n_{r}\left(d / d_{r}\right)^{2(\beta-l)}$ for areas and $n_{s}=n_{r}\left(d / d_{r}\right)^{3(\beta-l)}$ for volumes, whereas $n_{s}=n_{r}$ holds in the classical approach $(\beta=1)$ by Monge (Guyon and Troadec 1994).

A log-log histogram of numbers $N_{p}$ of pores with size $d_{p}$ yields a straight line section with slope $-3 \beta$, Figure $4 \mathrm{c}$. This corresponds to the major part of a skew stable Lévydistribution (Sato 1999). More precisely speaking, such a probability distribution comes close to a power-law in the falling branch. It is called stable since any sum of independent random values with such a distribution has the same kind of distribution. This uncommon central limit theorem holds strictly without cutoffs, but then mean values and higher moments diverge. There is no divergence with cutoffs, and random sums tend again to such distributions except for extremely big numbers (Mantegna and Stanley 1994). Such distributions can arise from successive fragmentation or agglomeration, e.g. sizes of grown-up animals from microbes to giants in habitats can exhibit them. 


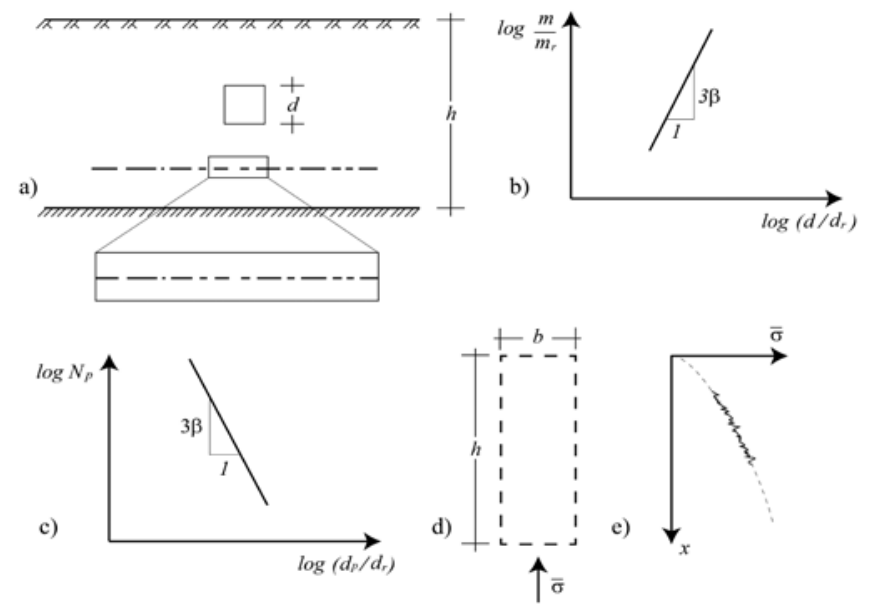

Figure 4: a) Layer with fractal pore system, b) solid mass of cubes, c) number of pores versus size, d) prism in the layer and e) vertical stress by weight versus depth

Vertical pressures by weight in fractally porous layers can be calculated by adding up the solid volumes of cubes, multiplying by specific gravity $g \rho_{s}$ and dividing by the base area. Figure $4 \mathrm{~d}$ shows that a prism of height $h$ and cross section $b^{2}$ has thus

$$
\sigma=g \rho_{s} n_{r} \frac{d_{r}^{3}}{b^{2}}\left(\frac{b}{d_{r}}\right)^{2 \beta}\left(\frac{h}{d_{r}}\right)^{\beta}
$$

The pressure $\sigma$ for a given depth decreases with increasing $b$, whereas without roughness $(\beta=1), \sigma=g \rho_{s} n_{s} h$, i.e. $\sigma$ is independent of $b$. The fractality $(\beta<1)$ requires a reference length $d_{r}$ and holds within cutoffs. Transition from addition of cubes to integration leads to the fractional integral

$$
\lambda=\frac{1}{\Gamma(\beta)} \int_{0}^{\xi}(\xi-\chi)^{\beta-1} \mu d \chi
$$

with $\lambda \equiv \sigma /\left(\rho_{s} n_{r} d_{r}\right), \xi \equiv x / d_{r}$ and $\mu=1$. Applying (2) for $x=x_{1}$ from 0 to $h$, and again for $x=x_{2}=x_{3}$ from 0 to $b$, leads to (1).

More generally speaking, the Riemann integral over a fractal distribution is equivalent to a fractional integral (Ren et al. 2003). The latter is a generalization of Cauchy's formula for multiple integrals to repeated integration of fractional degree $\alpha$ (Gorenflo and Mainardi 2000). It requires the Gamma function $\Gamma$ and dimensionless variables, e.g. by means of a reference length $d_{r}$. With fractal uniformity (1) and (2) indicate that the weight is no more extensive as without roughness ( $c f$. length and area in the Introduction). We call quantities fractionally extensive which would be extensive (in Rankine's sense) without spatial roughness, but they have to be expressed by fractional integrals with it. Cutoffs can be applied using expression (2) as well as (1) by keeping $b$ within $d_{\min }$ and $d_{\max }<h$.

The inversion of (2) reads $d^{\beta} \lambda / d \xi^{\beta}=\mu$ with a fractional derivative. This may be understood as a weighted average of secants for different section lengths, or as an objective gradient of a fractal distribution by means of the Gamma function and a reference length for normalization. This gradient is no more local in the classical continuum sense, but refers to a point in the fractional image of roughness. In the case considered here the fractal distribution represents the internal force-roughness by weight (Figure 4e). More precisely $D_{\alpha} \lambda$ with a specified operator $D_{\alpha}$ can be written instead of $d^{\alpha} \lambda / d \xi^{\alpha}$, this is needed for algebraic operations (Gorenflo and Mainardi 2000).

Turning to temporal fractality, we consider now a fractally uniform cubical range of jammed rock fractions. The latter may be grains or more densely packed pieces of rock. They constitute a perfect sample without deviations from fractal uniformity along the boundaries. We consider evolutions of spatial averages with time $t$, keeping in mind that spatial distributions are fractal so that classically extensive quantities are fractionally extensive as outlined above for weight. Imagine non-disturbing sensors which indicate seismicity, stress and shape of our cube. Figure 5 shows idealized plots of microseismic bursts and simultaneous sudden changes of stress and shape. This may be concluded from triaxial tests with dry hard-grained samples, confined by a membrane under constant pressure (Gudehus and Touplikiotis 2012). Slow axial loading causes audible microseismic bursts, and almost simultaneous changes of overall state and shape as long as the travel times of microseismic waves within our cube are much shorter than the intervals between seismic bursts.

Cube-averaged extensive properties $X_{i}$, in particular seismic energy, boundary forces (expressed by stress components) and lengths (changing by deformation) exhibit the same temporal fractality. Although the temporal 


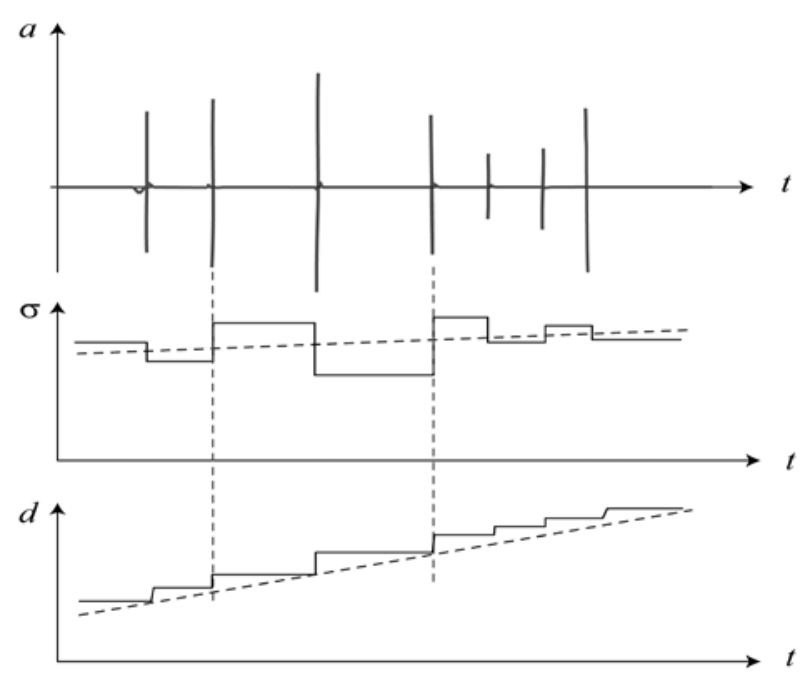

Figure 5: Schematic fractal evolution of averages of a cubical zone of jammed grains, a) seismicity, b) stress and c) shape

fluctuations have different intensities box-counting as in Figure 1, would thus yield the same fractal dimension. This justifies fractional balance equations

$$
\frac{d^{\alpha}\left(X_{i} / X_{i r}\right)}{d\left(t / t_{r}\right)^{\alpha}}=-\kappa_{i} X_{i} / X_{i r}+\mu_{i}
$$

with reference quantities $X_{i r}$ and $t_{r}$, a loss factor $\kappa_{i}$ and an input $\mu_{i} . X_{i}$ dwindles with constant $\kappa_{i}$ and $\mu_{i}=0$, monotonously for $0<\alpha<1$ and with oscillations for $1<\alpha$ $<2$. For a constant $\mu$ and $c a .0 .8<\alpha<1$ our $X_{i} / X_{i r}$ tends to $\mu$. For harmonic $\mu\left(t / t_{r}\right.$ ) the term $X_{i} / X_{i r}$ becomes periodic (harmonic for $\alpha=1$ or 2). Comparing such asymptotic solutions with observed attractors can serve to estimate $\alpha, \kappa_{i}$ and $\mu_{i}$ (Gudehus and Touplikiotis 2012). Expression (3) works only for increasing $t$, this may be attributed to causality for dissipative evolutions.

Balance equations like (3) are coupled by means of $X_{i-}$ dependent $\kappa_{i}$ and $\mu_{i}$. In an economic sense they express that the capital of market partners varies fractally with time by capital-dependent loss, and by input or output among each other and with the surroundings. For our idealized cube of jammed rock fractions coupled balances refer to shape and energies (elastic, seismic and thermal). This will be partly outlined later on and balances of extensive quantities will already be treated in the sequel. Coupled balances like (3) can be transformed to classical, i.e. non-fractional ones by means of fractional time stretching. This means that $t$ is transformed so that fractional integrals change into ordinary ones, and it is justified for the stable range with ca. $0.9<\alpha<1$.

Spatio-temporal balances of fractionally extensive quantities $X_{i}$ can in simple cases be written as

$$
\frac{\partial^{\alpha}\left(X_{i} / X_{i r}\right)}{\partial\left(t / t_{r}\right)^{\alpha}}=\kappa_{i} \frac{\partial^{\beta}\left(X_{i} / X_{i r}\right)}{\partial\left(x_{j} / d_{r}\right)^{\beta}}+\mu_{i}
$$

with reference quantities $X_{i r}, t_{r}, d_{r}$, and fractalities $\alpha, \beta$ as outlined further above and summation over $j$ for fractional gradients. Expression (4) represents a balance for a section of space-time with fractal distribution. The loss term with $\kappa_{i}$ does not need to be proportional to the fractional gradient of $X_{i}$ as written for simplicity in (4). In addition to shape and energies as for fractally uniform cubes, balanced quantities are mass and momentum, both are conserved for sums of constituents.

We assume isofractality, i.e. $\alpha=\beta$. This is justified by the same narrow range of $\alpha$ and $\beta$ and by the spatio-temporal coupling via microseismic elastic waves. The latter suggests $d_{r} / t_{r}=c_{s}$ with the velocity of elastic shear waves $c_{s}$ for isotropic states. With higher void ratios and lower pressures $\alpha$ decreases, which depends also on mineral and temperature. Apart from this moderate multifractality (see more in subsequent sections) one can transform (4) into non-fractional balances by means of a fractional spacetime stretching (Gudehus and Touplikiotis 2012). This is justified for the stable regime with the narrow range of $\alpha$.

\section{Attenuation and subordination}

As proposed by Gudehus (2010) for sand, we assume the specific elastic energy

$$
w_{e}=a h_{s} \Delta^{\nu}\left(1+\frac{b \Theta^{2}}{\Delta^{2}}\right)
$$

for jammed grains or rock fractions. Therein $\Delta$ and $\Theta$ denote invariant volumetric and deviatoric elastic deformations. The solid hardness $h_{s}$ and the exponent $v \approx 3$ depend on mineral and particle shape, the factors $a$ and $b$ depend on the porosity. Mean pressure and root mean square shear stress are energy- conjugated via $p=\partial w_{e} / \partial \Delta$ 
and $\tau=\partial w_{e} / \partial \Theta$. These first and second invariants can be related with components $\sigma_{i j}$ and $\epsilon_{i j}^{e}$, third invariants could also be taken into account.

Small deviations of stress and elastic strain from an equilibrium are captured by the hypoelastic relation

$$
\delta \sigma_{i j}=\frac{\partial^{2} w_{e}}{\partial \epsilon_{i j}^{e} \partial \epsilon_{k l}^{e}} \delta \epsilon_{k l}^{e}
$$

with summation in $\mathrm{kl}$. Using invariants this linearization implies the quadratic form

$$
\delta^{2} w_{e} \equiv \frac{\partial^{2} w_{e}}{\partial \Delta^{2}}(\delta \Delta)^{2}+2 \frac{\partial^{2} w_{e}}{\partial \Delta \partial \Theta} \delta \Delta \delta \Theta+\frac{\partial^{2} w_{e}}{\partial \Theta^{2}}(\delta \Theta)^{2}
$$

which describes second-order changes of $w_{e}$ for small variations of $\Delta$ and $\Theta$. Equilibrium states, i.e. $\delta w_{e}=0$ with $\delta^{2} w_{e}>0$, are stable as any deviation requires additional energy. Then $\delta^{2} w_{e}>0$ holds also with components, i.e. $\delta \sigma_{i j} \delta \epsilon_{i j}^{e}>0$ with summation in $i j$.

We consider now a fractally uniform zone with hypoelastic deviations from a stable equilibrium. Fractal uniformity means that stress $\sigma_{i j}$ and elastic strain $\epsilon_{i j}^{e}$ at equilibrium have no fractional gradients. Both have the same fractality due to $\sigma_{i j}=\partial w_{e} / \partial \epsilon_{i j}^{e}$. With isofractality as presented in the previous section, a small deviation of position $u_{i}$ implies a velocity

$$
\frac{v_{i}}{c_{s}}=\frac{\partial^{\alpha}\left(u_{i} / d_{r}\right)}{\partial\left(t / t_{r}\right)^{\alpha}}
$$

and the deformation

$$
\epsilon_{i j}=0.5\left[\frac{\partial^{\alpha}\left(u_{i} / d_{r}\right)}{\partial\left(x_{j} / d_{r}\right)^{\alpha}}+\frac{\partial^{\alpha}\left(u_{j} / d_{r}\right)}{\partial\left(x_{i} / d_{r}\right)^{\alpha}}\right]
$$

These are fractional representations of fractal distributions with dimensionless quantities for metric correctness.

The balance of momentum reads

$$
\frac{n_{r} \rho_{s} d_{r}}{h_{s}} \frac{\partial^{\alpha}\left(v_{i} / c_{s}\right)}{\partial\left(t / t_{r}\right)^{\alpha}}=\frac{\partial^{\alpha}\left(\delta \sigma_{i j} / h_{s}\right)}{\partial\left(x_{j} / d_{r}\right)^{\alpha}}
$$

with normalization as further above and summation in $j$. It expresses the conservation of momentum with fractality as by (4) without input as small deviations do not change the weight. Insertion of (6), (8) and (9) into (10) yields the fractional wave equation

$$
\frac{n_{r} \rho_{s} d_{r}}{h_{s}} \frac{\partial^{2 \alpha}\left(u_{i} / d_{r}\right)}{\partial\left(t / t_{r}\right)^{2 \alpha}}=\frac{\partial^{2}\left(w_{e} / h_{s}\right)}{\partial \epsilon_{i j}^{e} \partial \epsilon_{k l}^{e}} \frac{\partial^{2 \alpha}\left(u_{j} / d_{r}\right)}{\partial\left(u_{k} / d_{r}\right)^{\alpha} \partial\left(u_{l} / d_{r}\right)^{\alpha}}
$$

with summation in $j$ and $k l$. Without roughness, i.e. $\alpha=1$, and with $w_{e}=a \Delta^{2}+b \Theta^{2},(11)$ is reduced to the classical wave equation for isotropic linear elasticity. With $\alpha=1$, $w_{e}$ by (5) and $v=2.5$, expression (11) was analyzed by means of the classical ansatz $u_{i} \propto \exp \left(\omega t+k_{i} x_{i}\right)$, leading to realistic stress- and porosity-dependent wave velocities of granular samples in the stable range (Mayer and Liu 2010).

The vectorial fractional differential equation (11) is not given in the literature. Only scalar special versions, which can be derived by confining to isotropic states (Gudehus and Touplikiotis 2012), have been solved for certain initial and boundary conditions (Mainardi and Tomoirotti 1997). They reveal a hysteretic damping for the hypoelastic range which would be missed by spatio-temporal stretching so that (11) is simplified with $\alpha=1$ to the version of Mayer and Liu (2010). A novel solution of (11) was obtained with simultaneous Laplace and Fourier transformations and Green functions (Gorenflo et al. 2012). Other than without roughness, whereby a classical ansatz leads to an eigenvalue problem as mentioned above, a fractional eigenvalue problem is only obtained with integral transformations. For the stable range all eigenvalues are real so that propagations can occur. However, due to the fractality elastic waves are no more sinusoidal and dissipation occurs since any wave generates smaller irregular ones. Extended exponential functions, which were proposed by Mittag-Leffler 100 years ago, provide an orthogonal base.

Without fractality hypoelastic oscillations would last forever and could be decomposed into harmonics or eigenmodes. There are no harmonics with fractality, but with stability the wave equation dictates a characteristic autogeneous dwindling after an initial disturbance. It can be proven (Gorenflo et al. 2012) that the power-law spectrum of an initial seismicity experiences a downward and rightward shift in the log-log plot (Figure 6). The amplitudes fade with $t$ by power-laws as all wave fractions induce erratic oscillations with higher frequencies due to 
the fractal pore system. This kind of attenuation works as an autogeneous attractor for different initial seismicities and is characteristic of stable states with fractality. This works already for the hypoelastic range and without gradients. With them the attenuation is enhanced by radiation. With dissipative dislocations the attenuation after an initial small disturbance is faster than hypoelastic and leaves back a small change of position and state, whereas it would not cause any trace without roughness.

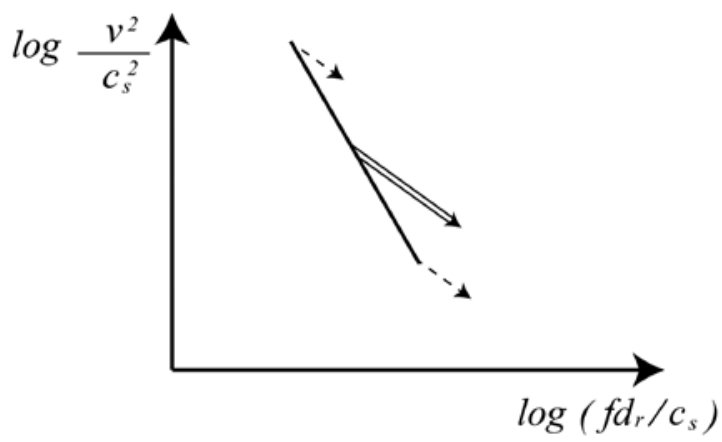

Figure 6: Initial seismic spectrum and shift (arrow) by attenuation in the stable range

A cubical fractally uniform zone of jammed grains may now be deformed monotonously from a stable state of rest. After a hypoelastic onset microseismicity arises with an intensity $T_{s}$ which may be named seismocrasy (Gudehus 2010). This name corresponds to the Greek 'thermocrasy' for temperature and is more suitable than 'granular temperature'. As without roughness the specific seismic energy is $w_{s} \propto T_{s}^{2}$ for assuring the stability of seismodynamic equilibria without elastic strain (Gudehus et al. 2010). For $T_{s}>0$ the elastic stress $\sigma_{i j}^{e}=\partial w_{e} / \partial \epsilon_{i j}$ deviates from the Cauchy stress $\sigma_{i j}=\left(1-\alpha_{s}\right) \sigma_{i j}^{e}$ with a $T_{s}$-dependent factor $0<\alpha_{s}<0.8$ as the grain contacts are jiggling. The elastic strain is augmented by overall stretching and reduced by seismically activated relaxation.

Better than with the classical calculus, the balance equations can be written as in (3) for temporal fractality (Gudehus and Touplikiotis 2012). They are coupled via $T_{s}$ and state that

- the seismic energy rises by average relative velocities of grains and dwindles by transition into heat,

- the elastic energy rises by changes of shape and dwindles by seismic relaxation.
Monotonous deformations with fractional rate $D$ lead to $T_{s} \propto D$ and to fractionally hypoplastic changes of $\sigma_{i j}$. This subordination, which is characterized by a monotonous exogeneos attractor (Gudehus 2010), works in the stable range. It can be reduced for non-fractional hypoplastic relations by fractional time-stretching (Gudehus and Touplikiotis 2012). Subordination implies rateindependence.

One may argue that the additional effort of using the fractional calculus is not worth it if it turns out that it is not needed. Well, not really, because the mechanical roughness is real and should not just be ignored. With the above argument the success of non-fractional models is further justified in spite of fractality for the stable range (but not otherwise, see next section). The argument works also for evolutions with pulsating seismicity in the stable range, i.e. for subordinations which imply pulsations of $T_{s}$. The behavior is then intermittently hypoelastic and hypoplastic with likewise rate-independent transitions. This kind of subordination is characterized by cyclic exogeneous attractors (Gudehus 2011). Fractional timestretching leads to non-fractional equations which produce realistic attractors (Gudehus et al. 2010). This means that hidden variables like back stress or intergranular strain for the internal force-roughness are not needed in energybased models in spite of the actual fractality. This helps in particular for cumulative effects (Gudehus 2010) and for rather fractal driven variations of $T_{s}$.

A kind of quantum seismodynamics is desirable for explaining the microseismicity during subordinations of cubical zones of jammed grains. The acoustic emission indicates seismons, i.e. seismic monads (Gudehus 2010), with a truncated power-law spectrum. These can be attributed to successive bursts of fractally distributed groups of grains which are jammed up to the stability limit. In the stable range seismons have randomly distributed polarizations, but other than with phonons for thermal oscillations the seismic energy is not conserved and not Gauss-like partitioned. The fractional Schrödinger equation

$e^{i \pi \alpha} \frac{\partial^{\alpha} \Psi}{\partial\left(t / t_{r}\right)^{\alpha}}=\frac{-2 m}{n_{r} \rho_{s} d_{r}^{3}} \frac{\partial^{2 \alpha} \Psi}{\partial\left(x_{i} / d_{r}\right)^{\alpha} \partial\left(x_{i} / d_{r}\right)^{\alpha}}+V\left(\frac{x_{i}}{d_{r}}\right)^{\alpha} \Psi$ 
yields the probability density $\Psi \Psi^{*}$ of seismons with mass $m$ and potential $V$. Like its precursor for particles (Laskin 2000), expression (12) yields truncated Lévy-spectra by means of Laplace and Fourier transformations. This suits to the stability of Lévy-distributions and the truncated fractality of microseismic bursts. The original Schrödinger equation, i.e. (12) with $\alpha=1$, yields much richer discrete spectra. The matter becomes more intricate with critical phenomena (next section).

Subordination in the large can occur in lithosphere sections, or in small substitutes at beaches or building sites or in model setups. It requires stability and excludes thermal activation (see last section). Coupled fractional balance equations such as (4) may then be reduced to nonfractional ones by fractional space-time stretching (as in previous section). This can suffice for the mild subcritical roughness with ca. $0.9<\alpha<1$ for suitably chosen regions. Relics of former critical phenomena (next section) cause an inevitable indeterminacy which is linked with rather vague notions such as fabric and inherent anisotropy. The indeterminacy can be reduced sometimes by means of attractors in the large (Gudehus 2011), which constitute a subordination in suitable soil regions.

The issue is more complex with rock and with bigger sections of the lithosphere. The mechanical roughness is apparently wilder for building sites and mines, this can be attributed to spatially variable cutoffs from previous critical phenomena and implies more indeterminacy than with soil. Fault patterns in the lithosphere are evidently multifractal and can lead to further critical phenomena (next section). Cases of coarse-graining may still correspond to subordination of the earth crust by magma convection below, but even in those cases seismic features of interest can at best be crudely estimated.

\section{Loss of stability and chain reactions}

Consider a fault system with a fractionally uniform zone at the verge of energetic convexity as shown in Figure $7 a$. This may represent a fractal fault zone with negligible fractional gradients at the verge of stability. One may imagine a zone in a fault system which is critical in a range with much smaller size than its depth. This specification is inevitably imprecise, an interpretation will be offered further below. Fractional uniformity means in particular that the stress tensor has the same alignment throughout our zone. Criticality with the simplest reasonable specific energy $w_{e}$ by (5) means that $w_{e}$ has a chair-point for the Coulomb condition $\tau^{2} / p^{2}=2 b /(v-2)$ (see Figure $7 b$ ). Then an increasing part of the elastic energy becomes kinetic for a one-sided section of elastic strain deviations.

a)
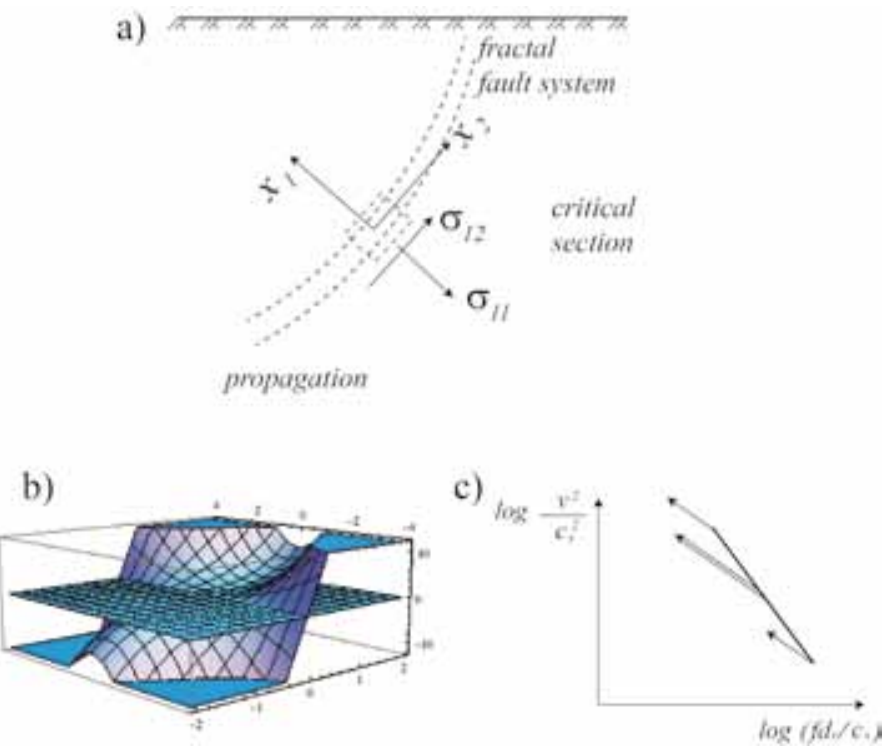

Figure 7: a) Critical zone of a fault system, b) elastic energy surface with tangent plane at the verge of convexity and c) spontaneous spectral shift of seismicity

The condition $\delta^{2} w_{e}=0$ by (7) can be translated into Cartesian stress and strain components. It means a linear dependence of the hypoelastic compliances, i.e. det $\partial^{2} w_{e} \partial \epsilon_{i j}^{e} \partial \epsilon_{k l}^{e}=0$. Thus the fractional wave equation (11) degenerates as propagations are impossible for the sector with $\partial^{2} w_{e}<0$. Instead, one-sided waves in this sector increase spontaneously at the expense of $w_{e}$. Combined Laplace and Fourier transformation with Green functions (Gorenflo et al. 2012) then produces a shift of an initial truncated power-law spectrum to the left and upwards as can be observed in Figure 7c. In other words, at a critical point an initial seismicity grows by the same factors for wave-length and -height throughout the wave package.

As for attenuations (previous section) the elastic strain components change with such a ratio that the loss of $w_{e}$ is maximal. With two invariants and $w_{e}$ by (5) this means that the spontaneous deviation from a chair point follows the steepest descent. This can be interpreted as maximal rate of entropy production due to the positive feedback of 
seismons (Gudehus 2010). The Lévy-spectrum exhibits a slight rightwards rotation of the log-log plot, but this kind of seismogenesis is still a stable Lévy-process. If the initial weak seismicity is not Lévy-like it is there with the named magnification so that one can speak of an autogeneous strange attractor (Gudehus 2011; Gorenflo et al. 2012).

The same spectral shift is also obtained with further degrees of freedom. The simplest model has only two invariants and two different stress components, one can incorporate a third invariant and a less symmetric stress state. One could extend the approach with polar quantities which arise from shear banding, this implies gradients which will be discussed below. One could also incorporate the extension of cracks by means of surface integrals (Budiansky and Rice 1973), and could include sorption energies for cracks (Rice 1978). The path-independent integrals enable energy balances for fractal crack systems, including critical points in the energetic sense of Griffith (1921). Fractional critical phenomena engulf thus the verge of energetic convexity and fractal distributions. This was addressed already in an overview paper on the mechanics of natural solids (Gudehus 2009), therein convexity was called concavity by considering an energy landscape from above, not from below as in mathematics and theoretical physics.

This approach is apparently insufficient as critical zones cannot exist with any extension due to their instability. One should instead imagine seismic reaction fronts which propagate to a certain extent. The front is fractal as with a forest fire or with irregular dominoes standing on a table. The reaction (e.g. burning or overturning) occurs in the front with a rather fuzzy extension, it leaves back a stable dead zone and propagates as long as a reaction in the zone ahead is triggered by the propagation. A chain reaction in a fractal fault zone propagates as long as the part ahead of the front is nearly critical so that the stress redistribution due to the collapse in the front leads to critical points immediately ahead.

One may try to extend Hadamard's concept of propagating discontinuities to fractional models of fractal evolutions with reaction fronts, but this will be mathematically difficult. Instead and less strictly, one can estimate the rate of propagation via a gradient-delayed collapse. This means that any critical zone has gradients of state towards its non-critical vicinity which reduce the rate of extension of seismic waves due to sidewards radiation (Gorenflo et al. 2012). The rate of extension can be reduced to zero, then the propagation is stuck. The upper cutoff bounds the rate of extension, this is an estimate of the propagation velocity of the seismic reaction front. This estimate is crude as it stems from a fractional image of a fractal reality, since gradients are fractional and can at best be estimated, as well as fractional balances for a reaction front are partly represented.

Consider real earthquakes for illustration. The 9.5 moment magnitude earthquake in Valdivia, Chile of 1960, is still the biggest ever recorded, involved roughly $20 \mathrm{~m}$ dislocation in a subduction zone of $c a .1000 \mathrm{~km}$ long and roughly $100 \mathrm{~km}$ deep within $c a .1000 \mathrm{~s}$. This means that the propagation of a seismic reaction front with up to $c a$. $1 \mathrm{~km} / \mathrm{s}$ horizontally and $100 \mathrm{~m} / \mathrm{s}$ vertically which came to an end farther off. In our fractional image a seismic chain reaction started somewhere near the surface and propagated with fractional speeds (in the sense of (8)) of the indicated order of magnitude. This represents a fractal reality, i.e. the extension of the critical zone and its propagation are rather fuzzy. The mechanism may be called a seismic soliton, in Mandelbrot's (1999) terms it means a transition from mild to wild roughness. The propagation could principally be recorded by suitably placed seismometers, and simulated by means of coupled fractional balances. Opaqueness and multifractality of the lithosphere delimit validations, but at least observed Lévy-spectra support our approach.

Data from other earthquakes could enable a sort of calibration, but this would be premature as our model should first be extended for further degrees of freedom (indicated above) and for thermal activation (next section). The Gutenberg-Richter rule confirms the Lévy-stability for any kind of superposition. The usual localization of active faults by means of P-waves (Hyndman and Hyndman 2009) could be improved by means of the actual fractality. Other than Burridge-Knopoff models (Cartwright et al. 1997), which are tectonically and tribologically over-simplified, our source mechanism is energetically sound and engulfs the real fractality. We concede that the real multifractality, which prevails due to earlier critical phenomena and arises 
again at critical points, is not yet properly captured. The narrow range $c a .0 .9<\alpha<1$, however, would require only a minor correction of balances. What counts more is the strong variation of cutoffs and minerals; both could principally be taken into account, but the lack of data will always require hypothetic completions.

A recent work by Silva Bustos (2008) may serve for illustration. A cross section with seismic sources indicates that a subduction is a fractal fault system so that its extension cannot be precisely determined (Figure 8). The depicted sources were localized from arrivals of waves in several earthquakes, but many minor sources cannot yet be localized. Thus the fractality of the subduction zone is but roughly represented. It appears that seismic solitons were stuck after running from the outcrop ca. $200 \mathrm{~km}$ downwards. It will be difficult to localize moving seismic sources because of the fractal indeterminacy.

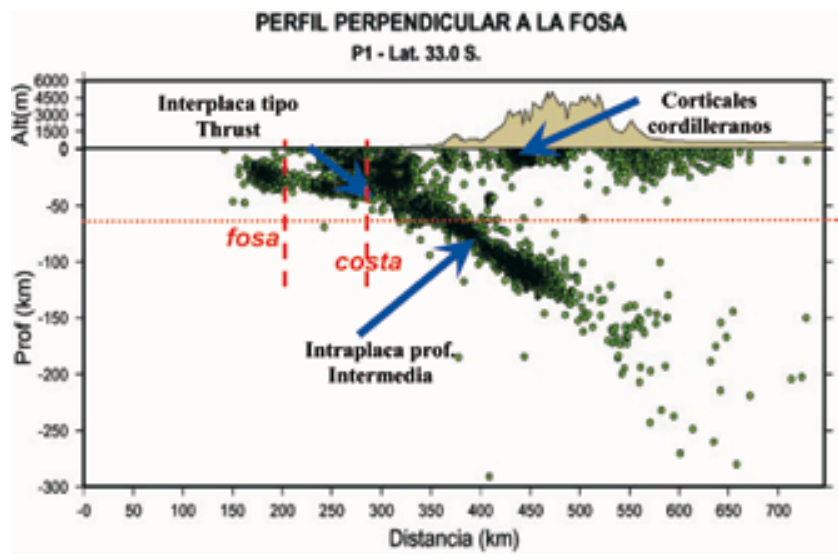

Figure 8: Cross section of Chile in the latitude $33^{\circ} \mathrm{S}$ with subduction and seismic sources (Silva Bustos 2008)

A $\log$-log-plot of number $N$ versus magnitude $M_{s}$ of seismic events shown in Figure 9, confirms the GutenbergRichter rule. It suits to the reproduction of Lévy-spectra by weighted additions as the seismic energy $E_{s}$ follows $\log E_{s}$ $\approx M_{s}+4.8$ empirically (Hyndman and Hyndman 2009). In other words, Gutenberg-Richter plots are a consequence of balaces with fractality and energetics at critical points (Gorenflo et al. 2012). The different slopes indicate a slight variation of fractality in our sense. This is not strictly valid as the usual determination of $M_{s}$ and $E_{s}$ is metrically imprecise with fractality, but could be improved by means of the fractional calculus. As indicated further above this argument holds also true with new ruptures which require a Griffith-type and not a Coulomb-type verge of convexity.

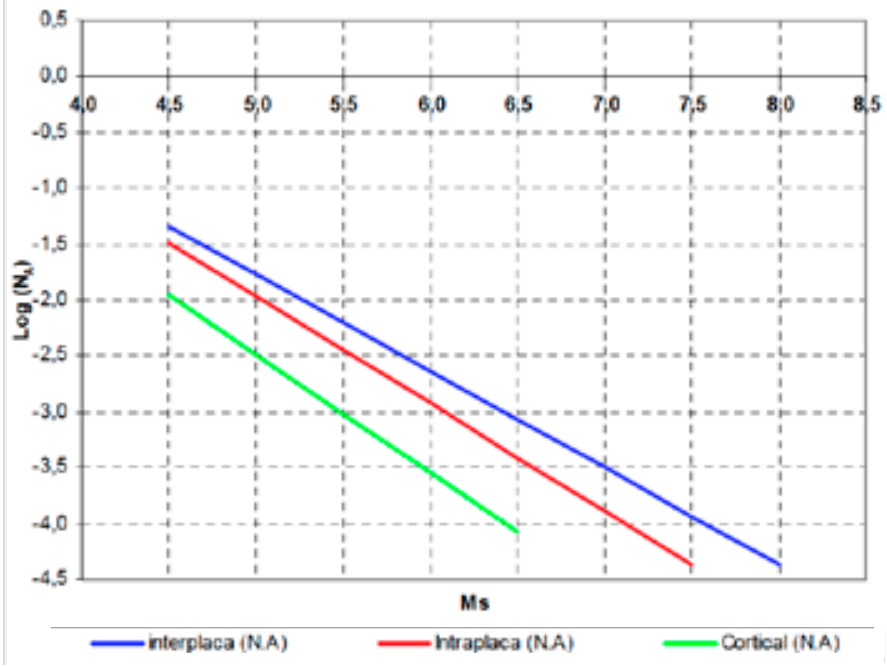

Figure 9: Seismicity of Chile in a Gutenberg-Richter plot (Silva Bustos 2008)

The lithosphere dynamics implies further critical phenomena than just seismic chain reactions. One is the original freezing and cracking, a second one is presumably related with the Moho discontinuity. Slow tectonics with formation and modification of faults is more complex if it is accompanied by earthquakes, and is multifractal anyway. Outbursts of pore fluid and gas mean cold volcanism, A.v. Humboldt noticed already its relation with faults. Bigger magmatic chain reactions $(\mu \epsilon \imath \mu \alpha=$ mixture) occur at hot spots of colliding plates, causing volcanoes and craters. Erosion and sedimentation imply critical phenomena with fluids. Man-made earthquakes occur if injections produce propagating critical zones which are delayed by diffusion, and similar effects occur after strong earthquakes (next section). A delicate issue is of course how recurrence times of natural earthquakes can thus increase.

The energetics at critical points means new territory in a physical and mathematical sense. The verge of convexity of the free energy is a general feature of the loss of stability, this holds from groups of molecules via conservative structures up to galaxies. An ensemble of molecules with Brownian motion exhibits then second-order phase transitions and arising fractality, this includes quantum effects (Binney 1992). Granular zones or formations of fissured rock are already fractal and become multifractal at critical points. The spontaneous growth of previously inactive degrees of 
freedom, typically related to shear banding or cracking, requires extensions of the fractional approach (indicated above). Balances of fractionally extensive quantities require fractionally extended differentials, this implies generalized seismic and thermal entropy productions. The quantum seismodynamics at critical points cannot be captured with (12) as for stability. It appears that two coupled fractional Schrödinger equations are needed as in quantum electrodynamics (Gudehus and Touplikiotis 2012).

\section{Seismic and thermal activations}

Deforming slowly, i.e. below $c a \cdot 10^{-3} / \mathrm{s}$, a cube of jammed rock fractions implies microseismic bursts with heatflashes. Thermography of dry sand samples revealed $T$ jumps at grain contacts beyond $1300^{\circ} \mathrm{K}$ within milliseconds (Luong 1982). Except for rapid deformation the heat is rapidly diffused after seismic flashes so that an increase of $T$ can hardly be observed. This holds also true for rock fractions in laboratory tests except for so high pressures $p$ that the diffusion of heat takes more time than its generation. The molecules at seismogeneus particle contacts are dislocated when past each other with higher rates by simultaneously higher $T$. The seismic activation is thus partly thermal due to $T$-rises within very short times (compared with encounter times) at minute mass fractions of the solid skeleton.

Seismic bursts in fractal fault zones can produce less localized $T$-jumps with overall deformation rates of around $10^{-3} \% / \mathrm{s}$ as thermal diffusion times can exceed heat production times. $T$-rises within active fault zones can thus engulf more than a minute fraction of all molecules. This can lead to softening and melting, but hardly to runaway instability (Vardoulakis 2002) and reactions (Hatzo et al. 2009). This type of co-seismic thermal activation is confined to temporarily seismogeneous zones, which are fractally distributed and exist only for short times in passing seismic reaction fronts (as seen in the previous section). More confined seismic bursts during subordinations cannot produce $T$-rises as encounter times of rock fractions exceed by far thermal diffusion times. Thus heat flashes due to seismic bursts characterize also lithosphere sections in a coarse-grained view.

Thermally activated dislocations with constant $T$ occur with rates which are proportional to $\exp \left(-E_{a} / k_{B} \mathrm{~T}\right)$ where $k_{B}$ is the Boltzmann constant. Dislocation units in aggregates of jammed rock fractions have activation energies $E_{a}$ from about $0.5 \mathrm{eV}$ for soft to $5 \mathrm{eV}$ for hard minerals at $\mathrm{T} \approx 270^{\circ} \mathrm{K}$ with $40 k_{B} \mathrm{~T} \approx 1 \mathrm{eV}$. Their thermal activation causes moderate or low rate-dependence or relaxation and/ or moderate or very slow creep. Thus lithosphere sections are more slowly jammed by tectonic deformations, without these jammed zones relax and pore systems close gradually. Critical points are therefore later or not attained with sufficiently slow or interrupted tectonic base shift. This thermal effect is evidently more marked with higher $T$ and lower $E_{a}$.

Pore fluids have $E_{a}<k_{B} T$, adsorbates of them have $E_{a}<10 k_{B} T$. After squeezing or injecting pore fluid this diffuses towards equilibrium, before the solid partial pressure $p_{s}=p-p_{f}$ is temporarily lower due to a higher fluid pressure $p_{f}$ (principle of effective stress). As the solid shear stress $\tau$ is thus not changed the solid skeleton can attain critical states so that the seismicity rises. Man-made earthquakes could thus increase the recurrence times of natural ones. Calculations require additional fractional balance equations for the pore fluid, coupled with the solid balances and with isofractality due to the fractal pore system and the seismicity. Again the roughness may be eliminated in the stable range by fractional stretching, but no more at critical points.

The interplay of thermal and seismic activations could approximately be followed up by separating mildly rough and rather aseismic slow periods from short seismic episodes with wild roughness. The latter are hardly influenced by thermal activation with uniform $T$ as seismic solitons pass by within short times. There is no diffusion of pore fluid during such episodes, but certainly in rest periods thereafter. Fractally distributed seismic bursts produce flash-like $T$-jumps, rapid dislocations can heat mylonite bands in faults so that reactions are enhanced. Other than assumed by some authors (Vardoulakis 2002; Hatzo et al. 2009), however, such reactions occur in jerks and can at best enhance the seismogenesis like fluid injections. The spontaneous generation of kinetic energy occurs again at critical points of the free energy, now including chemical potentials with capillary effects. The Lévy-type seismicity 
can only arise from fractality. This holds also true for the seismogenesis deeply below the lithosphere with spontaneous phase transitions in shear bands.

The solid-fluid interaction is the most important type of interplay of seismic and thermal activations. Except for methane cushions the fractal pore system is filled with almost incompressible water and dissolved minerals. This fluid experiences fractional diffusion (Gudehus and Touplikiotis 2012) with seepage and dwindling porosity in aseismic periods, then solubles can also condensate. Seismic episodes in critical zones are far shorter so that simultaneous diffusion and condensation are negligible. Then the pore pressure $p_{w}$ is higher or lower than the previous hydrostatic one as the porosity tends to decrease or increase, respectively (Gudehus 2011). The tendency for contractancy or dilatancy depends on the porosity, and on the ratio of mean solid pressure $p_{s}$ and hardness $h_{s}$. Similar changes of $p_{w}$ and $p_{s}$ are produced by injection or depletion of pore water.

The diffusion of pore water from a zone with seismogenesis by pumping in water causes a spreading of the zone with higher than hydrostatic $p_{w}$. As the total pressure $p=p_{s}+p_{w}$ and the deviatoric stress $\tau$ remain rather constant critical zones can arise in the neighbourhood if $p_{w}$ rises. This leads to delayed natural aftershocks or man-made earthquakes. It may suffice to estimate diffusion and related stress redistribution in the stable range without roughness, as this is mild and can be formally removed by a fractional stretching of spacetime, but this is no more legitimate at critical points. There is no way around the fractional calculus in case of seismogenesis, particularly with solidfluid coupling.

\section{Conclusions}

We conclude in particular that lithosphere sections have a fractal system of pores and internal forces. Balances of classically extensive properties require fractional integrals or fractional differential equations. Attenuation and subordination in the stable range imply Lévy-type seismicity can be captured with fractional calculus, but balances without this mild roughness may often suffice.

The loss of stability and seismogeneous chain reactions can only be captured with the fractional calculus and wild roughness arises alongside from multifractality. Seismic bursts imply heat flashes which can cause local softening and reactions in short episodes, whereas thermal activation causes slow relaxation and creep so that tectonic jamming is reduced. The water pressure in the pores rises in seismic episodes, this rise spreads by diffusion and can cause aftershocks, this can occur similarly after pumping in water.

The agenda for further research comprehends in particular, inherent and changing multifractality, fault systems with gradients and multifractality, Griffith-Rice fracture with fractality, coupling of solid and fluid with seismic and thermal activation, analytical bounds and numerical simulations and monitoring and control in situ.

\section{Acknowledgement}

Andrzej Niemunis, Mauro Poblete and Asterios Touplikiotis improved this paper by valuable hints and technical support, this is greatfully acknowledged.

\section{References}

Bak, P., Tang, C. and Wiesenfeld, K. (1987). Self-organized criticality: an explanation of 1/f noise. Physical Review Letters 59 (4): 381-384

Binney, J. (1992). The Theory of Critical Phenomena. Clarendon Press

Budiansky, B. and Rice, J.R. (1973). Conservation laws and energy release rates. Journal of Applied Mechanics 40, 201-203

Cartwright, J.H.E., Hernández-García, E. and Oreste Piro, O. (1997). Burridge-Knopoff Models as Elastic Excitable Media. Physical Review Letters 79, 527-30

Gorenflo, R. and Mainardi, F. (2000). Essentials of Fractional Calculus. Printed from MaPhySto Center, free online

Gorenflo, A., Gudehus, G. and Touplikiotis, A. (2012). On the stability of a fractal fault system. Zeitschrift für Angewandte Mathematik und Mechanik ZAMM (under preparation)

Griffith, A.A. (1921). The Phenomena of Rupture and Flow in Solids. Philosophical Transactions of the Royal Society of London A 221, 163-198

Gudehus, G. (2009). Mechanics of natural solids - a symposium 
in Horton, Greece. Obras y Proyectos 6, 4-7

Gudehus, G. (2010). Psammodynamics - Attractors and Energetics. 9th HSTAM Int. Congr. Mechan., Limassol, online

Gudehus, G. (2011) Physical Soil Mechanics. Springer, Berlin

Gudehus, G., Jiang, Y. and Liu, M. (2010). Seismo- and thermodynamics of granular solids. Granular Matter 13 (4), 319-340

Gudehus, G. and Touplikiotis, A. (2012). Clasmatic seismodynamics - Oxymoron or pleonasm?. Soil Dynamics and Earthquake Engineering 38, 1-14

Guyon, E. et Troadec, J.-P. (1994). Du sac de billes au tas de sable. Odile Jacob, Paris

Hatzo, Y., Sulem, J. and Vardoulakis, I. (2009). Meso-scale Shear Physics in Earthquake and Landslide Mechanics. Taylor and Francis

Heidbach, O.,Tingay, M. and Wenzel, F. (2010). Frontiers in Stress Research: Preface. Tectonophysics 482, 1-4

Hyndman, D. and Hyndman, D. (2009). Natural Hazards and Disasters. Chapter 3: Earthquakes and their causes. Brooks/ Cole: Cengage Learning, 2nd ed.

Laskin, N. (2000). Fractional quantum mechanics. Physical Review E 62/3, 3135-3145

Luong, M.P. (1982). Mechanical aspects and thermal effects of cohesionless soils under cyclic and transient loading. IUTAM Conf. Deform. Fail. Gran. Mat. Delft, 239-246

Mainardi, F. and Tomoirotti M. (1997). Seismic pulse propagation with constant $Q$ and stable probability distributions. Annali di
Geofisica XL 5, 1311-1328

Mandelbrot, B. (1982). The Fractal Geometry of Nature. W.H. Freeman, New York

Mandelbrot, B. (1999). Multifractals and 1/f Noise - Wild SelfAffinity in Physics. Springer, New York

Mantegna, R. and Stanley, H.E. (1994). Stochastic process with ultraslow convergence to Gaussian: The truncated Levy Flight. Physical Review Letters 73, 2946-2994

Mayer, M. and Liu, M. (2010). Propagation of elastic waves in granular solid hydrodynamics. Physical Review E 82, art. 042301

Ren, F.-Y., Liang, J.-R., Wang, X.-T. and Qiu, W.-Y. (2003). Integrals and derivatives on net fractals. Chaos, Solitons and Fractals 16, 107-117

Rice, J.R. (1978). Thermodynamics of the quasi-static growth of Griffith cracks. Journal of the Mechanics and Physics of Solids 26, Issue 3, 61-78

Sato, K.-I. (1999). Levy Processes and Infinitely Divisible Distributions. Cambridge University Press

Silva Bustos, N.A. (2008). Caracterización y determinación del peligro sísmico en la región metropolitana. Civil Eng. thesis, Universidad de Chile, online

Turcotte, D.L. (1997). Fractals and Chaos in Geology and Geophysics. 2nd ed., Cambridge University Press

Vardoulakis, I. (2002). Steady shear and thermal run-away in clayey gauges. International Journal of Solids and Structures 39, Issues 13-14, 3831-3844

Xie, H. (1933). Fractals in Rock Mechanics. Balkema, Rotterdam 